\title{
A Congestion-Aware Node Cooperation Mechanism based on Double Auction for Opportunistic Networks
}

\author{
Qingfeng Jiang ${ }^{1,2 *}$, Chaoguang Men ${ }^{1}$, Zeyu Tian ${ }^{1}$ and Meijuan Jia ${ }^{1,2}$ \\ ${ }^{1}$ College of Computer Science and Technology, Harbin Engineering University, \\ Harbin 150001, China \\ ${ }^{2}$ College of Computer Science and Information Technology, Daqing Normal \\ University, Daqing 163712, China \\ qingfeng_jiang@163.com
}

\begin{abstract}
In opportunistic networks, selfish nodes will refuse to forward messages for others to save their precious resources such as bandwidth, buffer and energy, and the system performance will degrade significantly. Therefore, it is necessary to motivate the selfish nodes to cooperatively forward messages. However, current cooperation mechanisms for opportunistic networks mainly focus on encouraging nodes to participate in message forwarding, but fail to consider the node congestion problem. When many messages are forwarded to the nodes with high connection degree, these nodes will become congested and discard most messages, which will seriously degrade the routing performance. To stimulate the selfish nodes with limited buffer to cooperatively forward messages, this paper proposes a congestion-aware node cooperation mechanism based on double auction, called CANCMDA. In CANCMDA, nodes first determine the self congestion degree according to current free buffer and message receiving speed. Then, combining message delivery probability and congestion degree, nodes trade messages based on double auction model. The double auction trade process is a bayesian game, and nodes get the optimal bid by solving the bayesian equilibrium to trade messages. The experimental results show that CANCMDA can effectively stimulate selfish nodes to cooperatively forward messages when congested, and achieves higher message delivery ratio with lower overhead ratio, compared with other mechanisms.
\end{abstract}

Keywords: Opportunistic networks, selfish, congestion, double auction, bayesian game

\section{Introduction}

Opportunistic networks are one of the most interesting evolutions of MANETs. In opportunistic networks, mobile nodes are enabled to communicate with each other even if a route connecting them never exists [1]. Possible application scenarios of opportunistic networks include wildlife monitoring, pocket switched networks, and bringing connectivity to rural areas etc.

There is often no complete path in opportunistic networks, so node communication in opportunistic networks is based on the store-carry-and-forward paradigm, i.e., a node needs to temporarily store and carry messages until encountering another node more suitable to bring the message to destination node. To deliver the messages effectively, many data forwarding and dissemination protocols such as Epidemic [2], Two-Hop [3], Binary Spray and Wait [4], Delegate [5], SimBet[6], ContentPlace[7], have been proposed. But the above protocols depend on the hypothesis that nodes are ready to forward messages for each other. The performance of these protocols will degrade seriously, when the selfish nodes refuse to forward messages for others, to save their precious resources such as energy, buffer, bandwidth etc. [8-9]. Therefore, it is imperative to design an effective node cooperation mechanism to achieve the cooperation among nodes. 
At present, some node cooperation mechanisms [10-17] have been proposed, but these mechanisms just focus on how to encourage nodes to deliver messages to other nodes with higher delivery probability, without considering the node congestion problem. Some researches [18,19] have shown that in forwarding routing, most messages will be forwarded to the nodes with high connection degree, resulting in the unbalanced load among nodes. Then the nodes with high connection degree will become congested and discard most of the messages due to the limited buffer, which will seriously degrade the routing performance. To stimulate the selfish nodes to cooperatively forward messages and reduce the impact of node congestion, we propose CANCMDA, a congestion-aware node cooperation mechanism based on double auction for opportunistic networks. In the mechanism, nodes calculate the message expectation values according to the message delivery probability and node congestion degree. Then nodes will trade the messages based on double auction to obtain more profits. By trading, messages of a node with high congestion degree will be transferred to another one with high delivery probability and low congestion degree, and finally to the destination node. The double auction trade process is a bayesian game, and nodes get the optimal bid by solving the bayesian equilibrium to trade messages, thereby achieving the cooperation.

The main contributions of this paper can be summarized as follows:

1) We propose CANCMDA, a congestion-aware node cooperation mechanism based on double auction for opportunistic networks.

2) We propose a way to calculate the message expectation value combining message delivery probability and congestion degree. A method to calculate the node congestion degree is also proposed according to current free buffer and message receiving speed.

3) We propose a double auction model for nodes to trade messages based on the message expectation value. The double auction process is a bayesian game, and the bayesian equilibrium is solved.

The rest of the paper is organized as follows. In Section 2, we review the related work. We present the system model in Section 3. We describe the cooperation mechanism CANCMDA in Section 4 and the simulation results of CANCMDA are given in Section 5. We conclude in Section 6.

\section{Related Work}

The problem of selfishness in opportunistic networks has attracted the attention of many researchers recently. In [8], the authors explore the impact of node cooperation on some representative routing algorithms of Epidemic, Two-Hop, Binary Spray and Wait for DTNs. It demonstrates a simple incentive mechanism that incorporates the cooperation degree can help improve the effectiveness of the three routing algorithms. In [9], the authors investigate how the selfish behavior of nodes affects the performance of DTN multicast. It demonstrates that the selfishness of not forwarding messages increases the message transmission delay and cost.

For the nodes selfishness, the authors in [10] propose a barter-based cooperation mechanism to increase the message delivery ratio in opportunistic networks. If a node misses any primary message, it can barter its secondary messages for primary messages with an encountered node, provided that they have complementary sets of primary and secondary messages. In [11], the authors propose a mechanism, called MuRIS, to allow nodes to cooperatively deliver information of interest to one another via chosen paths utilizing few transmissions. In [12], the authors present an incentive and privacy-aware data dissemination (IPAD) mechanism for opportunistic networks, not only to exploit how to protect mobile node's identity privacy, location privacy and social profile privacy, but also to provide a secure incentive for privacy-aware data dissemination. [10-12] focus on the data dissemination mechanism of one-to-many communication paradigm, different from the CANCMDA designed for the single-copy routing protocol. 
In [13], for the node selfishness, the authors propose a node cooperation mechanism Give2Get. In Give2Get, the forwarding node needs to provide the relay proof for the source node, if a message is dropped and the forwarding node cannot provide the relay proof, then the source node will notify the central authority and the forwarding node will be excluded from the system. In [14], the authors propose a credit-based incentive system, called MobiCent, to stimulate DTN selfish nodes to cooperatively forward messages for others. MobiCent also provides different payment mechanisms to cater to client that wants to minimize either payment or delivery delay. In [15], the authors propose two creditbased rewarding mechanisms, called earliest path singular rewarding mechanism and earliest path cumulative rewarding mechanism respectively, to ensure the nodes truthfully forward messages. The proposed rewarding mechanisms can prevent selfish nodes from having malicious behaviors, such as edge insertion attack, edge removal attack, and content modification attack. In [16], the authors propose a secure multilayer credit-based incentive mechanism Smart, to stimulate cooperation among DTN nodes. In Smart, a layered coins model is presented and serves as virtual currency to pay for the relays who participate in forwarding messages. In [17], the authors propose Multicent, a game theoretical incentive mechanism that not only provides cooperative incentive but also encourages nodes to follow defined rules to realize the desired performance objective.

The above cooperation mechanisms only motivate nodes to deliver messages to other nodes with higher delivery probability, without considering the node congestion problem. So we design a congestion-aware node cooperation mechanism based on double auction for opportunistic networks to improve the routing performance when nodes are congested.

\section{System Model}

As shown in Figure 1, the components of the system model are:

TTP (Trusted Third Party) as the central authority is responsible for issuing public and private keys and completing the verification and credit clearance service for mobile nodes.

Fixed Network, including Internet and wireless Access Points (AP), is responsible for connecting the mobile nodes and TTP. Mobile nodes can obtain public and private keys from the TTP via AP and Internet. When encountering the AP, the nodes will send the trade tickets to TTP by AP, then the TTP will achieve the credit clearance for mobile nodes.

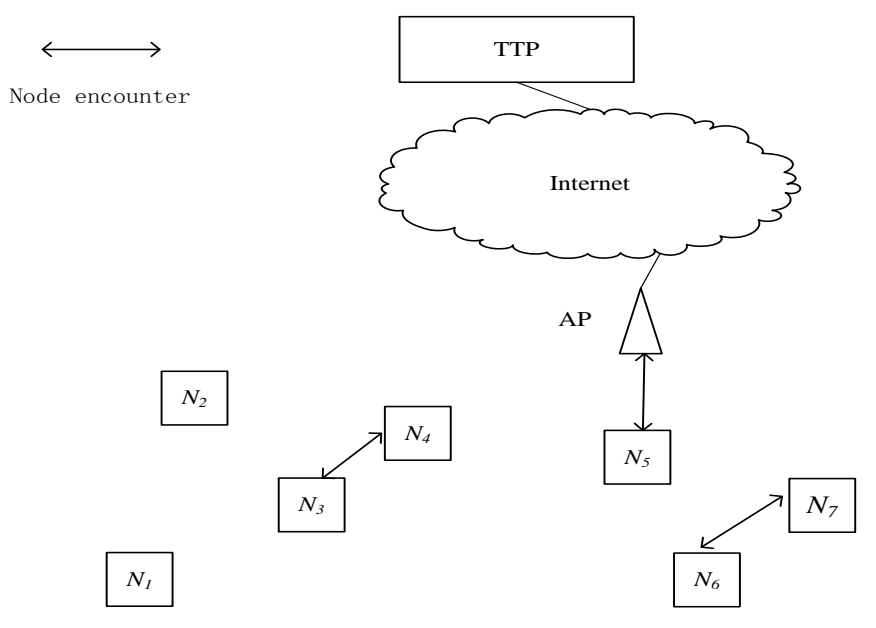

Figure 1. Overview of CANCMDA System Model

Mobile Nodes $N_{1}-N_{7}$ in Figure 1 denotes some pedestrians who carry the equipments with the function of Bluetooth and WIFI. The mobile nodes send the messages such as 
traffic and location to others. When a message is delivered to the destination node, the destination node will pay one credit for the last forwarding node. Because a node will pay some credits when receiving messages, it has to forward messages for other nodes to obtain more credits when having enough resource.

\section{Cooperation Mechanism based on Double Auction}

\subsection{Cooperation Mechanism Overview}

When two nodes encounter, they calculate the message expectation values for a message according to the message delivery probability and node congestion degree. If a node's message expectation value is smaller, it can sell the message to the other node with larger expectation value. By the trading, the node with high congestion degree can send the message to the node with high delivery probability and low congestion degree, to prevent the message from being dropped.

Assuming nodes $N_{i}$ and $N_{j}$ encounter, the congestion-aware cooperation mechanism based on double auction is shown in Figure 2, it includes the following steps:

1) Both nodes exchange message lists $L_{i}, L_{j}$ for each other.

2) For the message $m_{i}$ in list $L_{i}$ of node $N_{i}$, nodes $N_{i}$ and $N_{j}$ calculate the message expectation values for message $m_{i}$ according to the message delivery probability and congestion degree respectively. If the message expectation value of $N_{j}$ is larger than $N_{i}$, then nodes $N_{i}$ and $N_{j}$ will trade $m_{i}$ by the double auction model. The process of message $m_{j}$ in list $L_{j}$ of node $N_{j}$ is same as message $m_{i}$.

3) If the trade is succeeded, then message $m_{i}$ is added to the send set $S e n d S_{i}$, otherwise another message will be traded. The trade of messages in node $N_{j}$ is same.

4) Nodes send messages to each other in accordance with the message sequence in send set $\operatorname{Send}_{i}$ and $\operatorname{Send} S_{j}$.

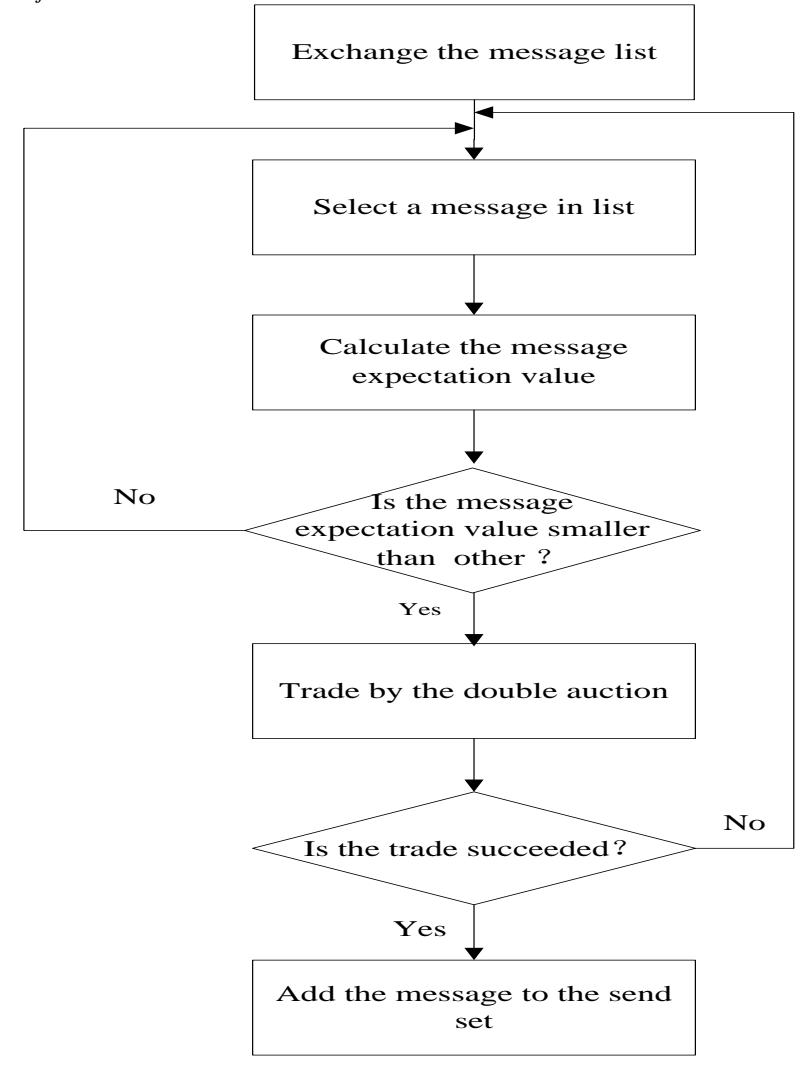

Figure 2. CANCMDA Mechanism Flow Chart 
When a message is successfully delivered to a buyer node, the buyer node will generate a trade ticket as in Figure 3, and encrypt it with its private key, then give it to the seller node. When the seller node encounters the AP, it will send the trade ticket to TTP by AP, and obtain its corresponding credits.

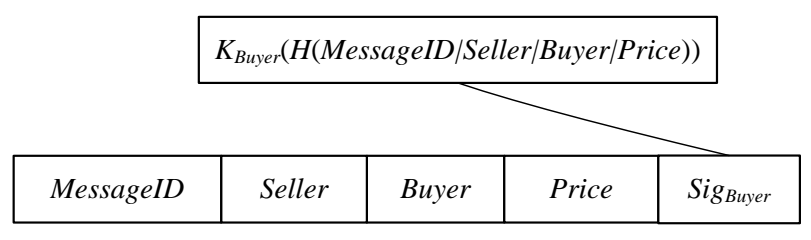

Figure 3. Trade Ticket

In the trade ticket, MessageID is the message identifier, Seller is the seller node, Buyer is buyer node, Price is the trade price, Sig $_{\text {Buyer }}$ is the digital signature of buyer node, $K_{\text {Buyer }}$ is the private key of buyer node, and $H$ is the hash function to generate the summary to ensure the integrity of message.

\subsection{Message Expectation Value Calculation}

When trading message, nodes need to calculate the message expectation value by message delivery probability and node congestion degree. Only when the message expectation value of seller is smaller than buyer, they can trade the message. The message expectation value is determined by the delivery probability and node congestion degree. The lower the delivery probability is, the smaller the message expectation value is. When the node congestion degree is higher, message has higher probability of being dropped due to buffer overflow, so the message expectation value will be smaller. Assuming that the destination node of message $m$ in node $N_{i}$ is node $N_{d}$, then the expectation value $V$ of message $m$ is calculated by Eq.(1).

$$
V=(1-\varepsilon) \times P_{i d}+\varepsilon \times(1-\text { ConDegree }), 0 \leq \varepsilon \leq 1
$$

where $P_{i d}$ is the delivery probability of node $N_{i}$ for message $m$, which has relation with the message remain time and encounter time between nodes $N_{i}$ and $N_{d}$, ConDegree is the node congestion degree before message $m$ is transferred and expired, $\varepsilon$ is the weight of node congestion degree, which is calculated as follow:

$$
\varepsilon=\text { ConDegree } /(\text { ConDegree }+P \text { id })
$$

When ConDegree is larger, the node will be more congested, and then the weight $\varepsilon$ is greater.

It can be seen that the message expectation value will be larger, when having higher message delivery probability and smaller congestion degree.

The process of calculating the message delivery probability $P_{i d}$ and node congestion degree ConDegree is as follows:

1) Calculating the message delivery probability $P_{i d}$

As [20,21], we also assume that the nodes encounter time follows an exponential distribution with mean value $1 / \lambda_{i d}$, where $\lambda_{i d}$ represents the contact frequency between nodes $N_{i}$ and $N_{d}$. The contact frequency $\lambda_{i d}$ between nodes $N_{i}$ and $N_{d}$ can be calculated by the following time average method:

$$
\lambda_{i d}=n / \sum_{l=1}^{n} t_{i d}^{l}
$$


where $t_{i d}^{1}, t_{i d}^{2}, \ldots, t_{i d}^{n}$ are encounter time samples between nodes $N_{i}$ and $N_{d}$. Thus, the Probability Distribution Function (PDF) of the encounter time $t_{i d}$ between nodes $N_{i}$ and $N_{d}$ can be expressed as Eq.(4).

$$
f\left(t_{i d}\right)=\lambda_{i d} e^{-\lambda_{i d}} t_{i d}
$$

Therefore, for the message $m$ of node $N_{i}$, if the destination node is node $N_{d}$ and the remaining valid time is $t_{\text {remain }}$, then the delivery probability of node $N_{i}$ for message $m$ is expressed as Eq.(5).

$$
P_{i d}\left(t_{\text {remain }}\right)=P\left(t_{i d} \leq t_{\text {remain }}\right)=\int_{0}^{t_{\text {remain }}} f\left(t_{i d}\right) d t_{i d}=1-e^{-\lambda_{i d} \times t_{\text {remain }}}
$$

The remaining valid time $t_{\text {remain }}$ is calculated as:

$$
t_{\text {remain }}=\mathrm{TTL}+t_{\text {create }}-t_{\text {current }}
$$

where TTL is the lifetime of message $m, t_{\text {create }}$ is the message generation time, $t_{\text {current }}$ is the current time when nodes $N_{i}$ and $N_{j}$ encounter.

\section{2) Calculating the node congestion degree ConDegree}

The node congestion degree ConDegree is calculated by Eq.(7).

$$
\text { ConDegree }= \begin{cases}0, & \text { BufFree }_{t}>\text { BufTh } \\ 1-\text { BufFree }_{t} / \text { BufTh }, & 0<\text { BufFree }_{t} \leq \text { BufTh } \\ 1, & \text { BufFree }_{t}<0\end{cases}
$$

where BufFreet is the free buffer size when message $m$ will be transferred to the destination node and TTL is expired, BufTh is the threshold of free buffer. When

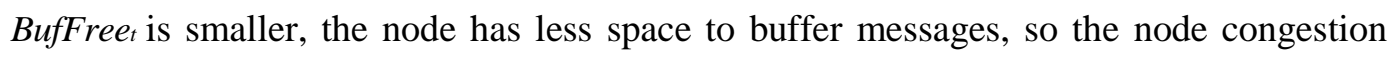
degree ConDegree will be higher.

The value of BufFreet is calculated as :

$$
\text { BufFree }_{t}=\text { BufFreecurrent }-R \times t
$$

where BufFree $_{\text {current }}$ is the current free buffer size when nodes $N_{i}$ and $N_{j}$ encounter, $R$ is the message receiving speed, $t=\min \left(1 / \lambda_{\text {id }}\right.$, tramain $), 1 / \lambda_{\text {id }}$ denotes the mean encounter time between nodes $N_{i}$ and $N_{d}, t_{\text {remain }}$ is the message remaining valid time. When the message receiving speed $R$ is larger, many messages will come into the buffer, then BufFreet will be smaller.

When calculating the message receiving speed $R$, the time is divided to many periods by period $T$, then the value of $R$ in a period $T$ is expressed as:

$$
R=(\text { BufFreebegin }- \text { BufFreeend }) / T
$$

where BufFree $_{\text {begin }}$ is the free buffer size at the beginning time of period $T$, and BufFree $_{\text {end }}$ is the free buffer size at the end time of period $T$. To restrain the effect of 
temporary burst, the new message receiving speed $R$ is calculated by the average of the current three successive messages receiving speed.

\subsection{Message Trade between Nodes}

4.3.1. The Message Auction Model: When encountering, two nodes will trade the messages by the double auction model according to message expectation values. Assuming that the seller and buyer bid Bidsell $\left(V_{\text {sell }}\right)$ and $B i d_{b u y}\left(V_{\text {buy }}\right)$ according to the message expectation values $V_{\text {sell }}, V_{\text {buy }}$ respectively. As in[22], if Bidbuy $\geq B i d_{s e l l}$, then the seller and buyer will trade the message by the final price of Price $=k B i d_{s e l l}+(1-k)$ Bidbuy $_{\text {, }} 0 \leq k \leq 1$, otherwise, no trade is made. Assuming that the seller and buyer do not know each other's real message expectation value, i.e., the message expectation value is private information, and the expectation value $V$ follows a uniform distribution $F(V)$ on the interval $[0,1]$, where $F(V)$ is the cumulative distribution function. Then the double auction is a static game of incomplete information, i.e., bayesian game. The model of the bayesian game[23] is as:

Players : seller, buyer

States : The set of all profiles ( $V_{\text {sell }}, V_{\text {buy }}$ ) of values, where $0 \leq V_{\text {sell }} \leq 1,0 \leq V_{\text {buy }} \leq 1$.

Actions : Each player's set of actions is the set of possible bids (nonnegative numbers).

\section{Signals :}

The set of signals that each player may observe is the set of possible values. The signal function $T_{\text {sell }}$ of player seller is given by $T_{\text {sell }}\left(V_{\text {sell }}, V_{\text {buy }}\right)=V_{\text {sell }}, T_{\text {buy }}$ of player buyer is $T_{\text {buy }}$ $\left(V_{\text {sell }}, V_{\text {buy }}\right)=V_{\text {buy }}$ (each player knows her own value $)$.

\section{Beliefs :}

Player seller assigns probability $F\left(V_{b u y}\right)$ to the event that the value of player buyer is at most $V_{b u y}$. Player buyer assigns probability $F\left(V_{\text {sell }}\right)$ to the event that the value of player seller is at most $V_{\text {sell }}$.

\section{Payoff functions :}

Player seller's payoff is $U_{\text {sell }}=k B i d_{\text {sell }}+(1-k)$ Bidbuy $-V_{\text {sell }}$, if Bidbuy $\geq B i d_{\text {sell }}$, and 0 if Bidbuy $<$ Bidsell . Player buyer's payoff is $U_{b u y}=V_{b u y}-\left(k B i d_{s e l l}+(1-k) B i d_{b u y}\right)$, if $B i d_{b u y} \geq B i d_{s e l l}$, and 0 if Bidbuy $<$ Bidsell .

In the bayesian game, the strategy $\operatorname{Bid} d_{\text {sel }}\left(V_{\text {sell }}\right)$ of seller is the function of message expectation value $V_{\text {sell }}$, the strategy $\operatorname{Bidbuy}\left(V_{b u y}\right)$ of buyer is the function of $V_{b u y}$. The strategy combination $\left(\operatorname{Bid}_{\text {sell }}^{*}\left(V_{\text {sell }}\right), \operatorname{Bid}_{b u y}^{*}\left(V_{b u y}\right)\right)$ is an optimal strategy if and only if it satisfies the following two conditions:

1) Seller optimal. Bid sell $\left(V_{\text {sell }}\right)$ is one solution to the optimization question in Eq.(10).

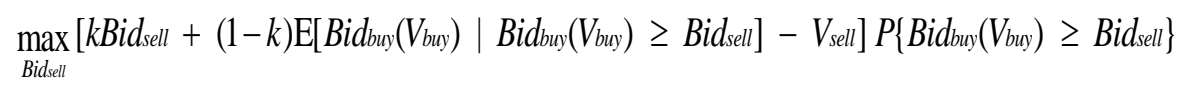

$\mathrm{E}\left[\operatorname{Bid}_{b u y}\left(V_{b u y}\right) \mid \operatorname{Bid} d_{b u y}\left(V_{b u y}\right) \geq \operatorname{Bid} d_{s e l l}\right]$ is the expectation bid of buyer, when the seller's bid is lower than buyer. $P\left\{\operatorname{Bidbuy}\left(V_{b u y}\right) \geq B i d_{s e l l}\right\}$ is the probability that $\operatorname{Bidbuy}\left(V_{b u y}\right)$ is greater than or equal to Bidsell .

2) Buyer optimal. $B d_{b u y}^{*}\left(V_{b u y}\right)$ is one solution to the optimization question in Eq.(11).

$$
\max _{\operatorname{Bidhuy}}\left[\operatorname{Bid} d_{b u y}-\left((1-k) \operatorname{Bid} b_{b u y}+k \mathrm{E}\left[\operatorname{Bid} d_{\text {sell }}\left(V_{\text {sell }}\right) \mid \operatorname{Bid} b_{b u y} \geq \operatorname{Bid} d_{\text {sell }}\left(V_{\text {sell }}\right)\right]\right)\right] P\left\{\operatorname{Bid} d_{b u y} \geq \operatorname{Bidsell}\left(V_{\text {sell }}\right)\right\}
$$


$\mathrm{E}\left[\operatorname{Bid}_{\text {sell }}\left(V_{\text {sell }}\right) \mid \operatorname{Bid}_{\text {buy }} \geq \operatorname{Bid} d_{\text {sell }}\left(V_{\text {sell }}\right)\right]$ is the expectation bid of seller, when the seller's bid is lower than buyer. $P\left\{B i d_{b u y} \geq B i d_{s e l l}\left(V_{\text {sell }}\right)\right\}$ is the probability that Bidbuy is greater than or equal to $\operatorname{Bid}_{\text {sell }}\left(V_{\text {sell }}\right)$.

4.3.2. Solving the Bayesian Equilibrium of Double Auction Model: Assuming the seller and buyer adopt the line bid strategy in Eqs.(12) and Eq.(13).

$$
\begin{aligned}
& \operatorname{Bid}_{\text {sell }}\left(V_{\text {sell }}\right)=\alpha_{\text {sell }}+\beta_{\text {sell }} V_{\text {sell }} \\
& \operatorname{Bidbuy}\left(V_{\text {buy }}\right)=\alpha_{b u y}+\beta_{\text {buy }} V_{b u y}
\end{aligned}
$$

\section{1) The process of solving the optimal bid Bidsell of seller is as:}

$V_{b u y}$ follows a uniform distribution of $[0,1]$, so $\operatorname{Bidbuy}\left(V_{b u y}\right)$ follows a uniform distribution of $\left[\alpha_{b u y}, \alpha_{b u y}+\beta_{b u y}\right]$, then in Eq.(10), it can be concluded that :

$$
\begin{gathered}
P\left\{\operatorname{Bid} d_{b u y}\left(V_{b u y}\right) \geq B i d_{s e l l}\right\}=\frac{\alpha_{b u y}+\beta_{b u y}-B i d_{s e l l}}{\beta_{b u y}} \\
\mathrm{E}\left[\operatorname{Bid} d_{b u y}\left(V_{b u y}\right) \mid \operatorname{Bid} d_{b u y}\left(V_{b u y}\right) \geq B i d_{s e l l}\right]=\frac{\frac{1}{\beta_{b u y}} \int_{B i d_{s e l l}}^{\alpha_{b u y}+\beta_{b u y}} x d x}{P\left\{B i d_{b u y}\left(V_{b u y}\right) \geq B i d_{s e l l}\right\}}=\frac{1}{2}\left(B i d_{s e l l}+\alpha_{b u y}+\beta_{b u y}\right)
\end{gathered}
$$

Substituting Eqs.(14) and (15) into the objective function of seller in Eq.(10), then it can be concluded that :

$$
\max _{\text {Bidsel }}\left[k B i d_{\text {sell }}+\frac{1-k}{2}\left(\text { Bid }_{\text {sell }}+\alpha_{\text {buy }}+\beta_{\text {buy }}\right)-V_{\text {sell }}\right] \frac{\alpha_{b u y}+\beta_{b u y}-B i d_{\text {sell }}}{\beta_{b u y}}
$$

Differentiating the Eq.(16) with respect to Bidsell yields the optimal bid of seller:

$$
\text { Bid }_{\text {sell }}=\frac{k}{1+k}\left(\alpha_{b u y}+\beta_{b u y}\right)+\frac{1}{1+k} V_{\text {sell }}
$$

\section{2) The process of solving the optimal bid Bidbuy of buyer is as:}

$V_{\text {sell }}$ follows a uniform distribution of $[0,1]$, so Bidsell $\left(V_{\text {sell }}\right)$ follows a uniform distribution of $\left[\alpha_{\text {sell }}, \alpha_{\text {sell }}+\beta_{\text {sell }}\right]$, then in Eq.(11):

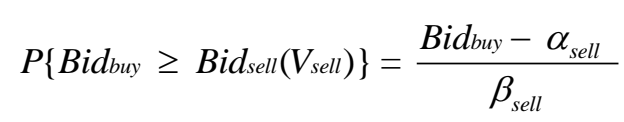

$\mathrm{E}\left[\operatorname{Bid}_{\text {sell }}\left(V_{\text {sell }}\right) \mid \operatorname{Bid} d_{\text {buy }} \geq \operatorname{Bid}\right.$ sell $\left._{(}\left(V_{\text {sell }}\right)\right]=\frac{\frac{1}{\beta_{\text {sell }}} \int_{\alpha_{\text {sell }}}^{\text {Bidduy }} x d x}{P\left\{\operatorname{Bid} d_{\text {buy }} \geq \operatorname{Bid} d_{\text {sell }}\left(V_{\text {sell }}\right)\right\}}=\frac{1}{2}\left(\alpha_{\text {sell }}+\operatorname{Bid}\right.$ buy $)$

Substituting Eqs.(18) and (19) into the objective function of buyer in Eq.(11), then : 


$$
\max _{\text {Bidhy }}\left[V_{\text {buy }}-\left((1-k) \text { Bidbuy }+\frac{1}{2} k\left(\alpha_{\text {sell }}+\text { Bidbuy }\right)\right)\right] \frac{\text { Bidbuy }-\alpha_{\text {sell }}}{\beta_{\text {sell }}}
$$

Differentiating the Eq.(20) with respect to Bidbuy yields the optimal bid of buyer:

$$
\text { Bidbuy }=\frac{k-1}{k-2} \alpha_{\text {sell }}-\frac{1}{k-2} V_{b u y}
$$

Combining Eqs.(12), (13), (17) and (21), the optimal bids of seller and buyer are as follows:

$$
\begin{gathered}
\text { Bidsell }=\frac{k}{2}+\frac{1}{1+k} V_{\text {sell }} \\
\text { Bidbuy }_{1}=\frac{(k-1) k}{2(k-2)}+\frac{1}{2-k} V_{b u y}
\end{gathered}
$$

Theorem 1: For a message, if the seller and buyer can trade successfully, then the message expectation values $V_{\text {sell }}$ and $V_{b u y}$ must satisfy the condition $V_{b u y}-\frac{2-k}{1+k} V_{\text {sell }} \geq \frac{k}{2}$.

Proof : If a message is traded successfully, then:

$$
\text { Bidbuy } \geq \text { Bidsell }
$$

$$
\begin{aligned}
& \stackrel{\text { Eqs.(22)(23) }}{\Rightarrow} \frac{(k-1) k}{2(k-2)}+\frac{1}{2-k} V_{b u y} \geq \frac{k}{2}+\frac{1}{1+k} V_{\text {sell }} \\
& \Rightarrow \quad V_{\text {buy }}-\frac{2-k}{1+k} V_{\text {sell }} \geq \frac{k}{2}
\end{aligned}
$$

It is the end of proof.

\section{Performance Evaluation}

\subsection{Simulation Setup}

We implement CANCMDA on the Opportunistic Networking Environment simulator [24], and evaluate its performance in the data sets of Infocom 05[25] and Cambridge 06 [26]. Infocom 05 is a data set collected during the Infocom 2005 student workshop. It contains 41 devices and covers approximately 3 days. The iMotes are programmed to log contacts of all visible mobile devices and a number of external devices which are not used in this paper. Cambridge 06 is a data set collected by distributing Intel iMotes to students of Cambridge university. This data set contains 36 nodes and covers 11 days. To be fair, the $k$ in the final price is $1 / 2$, so the buyer and seller can carry equal weights in determining the final price. In Eq.(7), BufTh is 0.1 times of total buffer size. More than $70 \%$ of the nodes' encounter times are not greater than one hour in Infocom 05 and 12 hours in Cambridge 06[27], so in Eq.(9), we set $T=\min (1 h$, TTL), $T=\min (12 h$, TTL) for the two data sets respectively. Every node has an initial credit of 100, and pays 1 credit for each delivered message. Further details on the default setup are shown in Table 1. 
Table 1. Simulation Parameters

\begin{tabular}{|c|c|}
\hline Parameter & Setting \\
\hline Simulation time & Infocom05:274883s; Cambridge06:964416s \\
\hline Warm up time & $30000 \mathrm{~s}$ \\
\hline Tail time & $30000 \mathrm{~s}$ \\
\hline Message generation interval & $5 \sim 15 \mathrm{~s}$ \\
\hline Message size & $1 \mathrm{MB}$ \\
\hline Buffer size & Infocom05: 80MB; Cambridge06:200MB \\
\hline Data transmission speed & $250 \mathrm{kBps}$ \\
\hline
\end{tabular}

\subsection{Performance Comparison}

CANCMDA is compared with the Direct routing mechanism where each node only stores its own messages, but does not forward messages for others, Smart mechanism[16] and Multicent mechanism[17]. The performance metrics of delivery ratio, delivery delay, overhead ratio and cumulative utility are introduced to evaluate the routing performance. The delivery ratio is defined as the ratio of successful delivered messages and total generated messages in the network; delivery delay is the average delivery delays of messages delivered to the destination node; overhead ratio is defined as the ratio of the total number of forwarding messages and the number of successfully delivered messages, which denotes how much times forwarding is needed to successfully deliver a message. Cumulative utility is the total credits obtained by a node, including the credits to pay for the delivered messages. We evaluate the impact of buffer size, TTL, and message generation interval on performance.

5.2.1. Effect of Buffer Size: As shown in Figure 4, the delivery ratio increases as the buffer size increases for all the 4 routing mechanisms. This is because nodes can carry more messages with larger buffer, and subsequently deliver more messages successfully. The delivery ratio of Direct is lowest, this is because each node only stores its own messages, and cannot forward the messages to others with higher delivery probability. As a result, some messages are dropped due to fullness of buffer or expiration of TTL. The delivery ratio of CANCMDA is higher than Smart and Multicent mechanisms. This is because Smart and Multicent cooperation mechanisms only encourage nodes to deliver the messages to other nodes with higher delivery probability, without considering the congestion problem. Many messages will be dropped when the receiving node has no free buffer. In CANCMDA, when the receiving node is congested, it will decrease the message expectation value according to the congestion degree and reduce the probability of trading successfully. Then the messages will be transferred to the nodes less congested, and avoid being dropped, so the message delivery ratio of CANCMDA is higher than other mechanisms. 


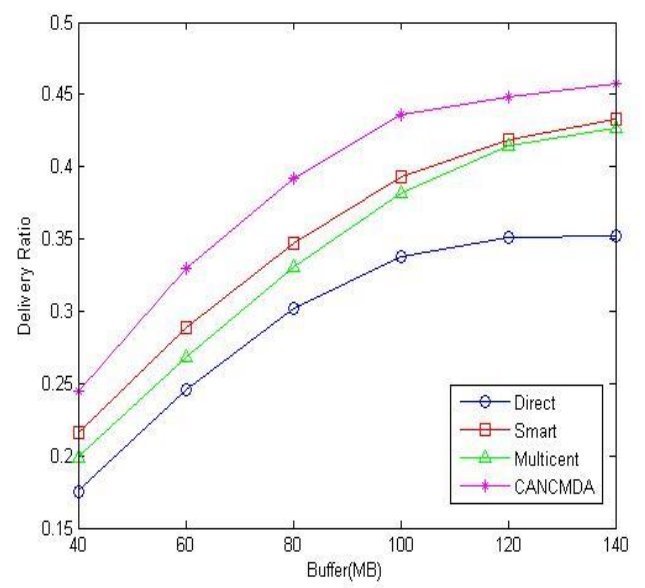

(a)

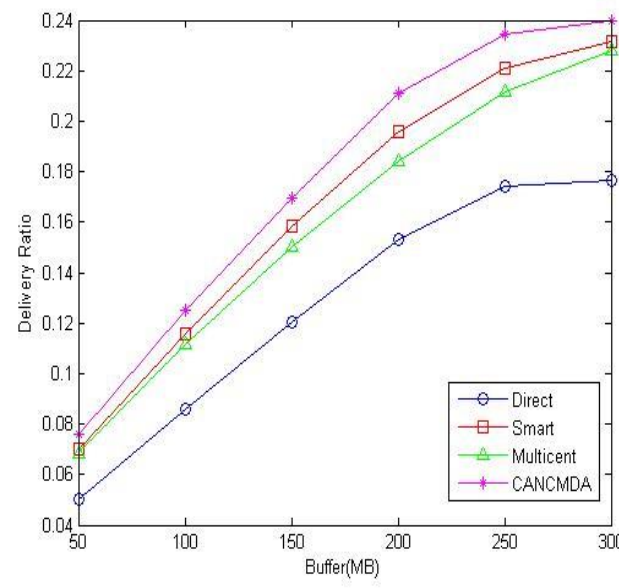

(b)

Figure 4. Delivery Ratio for Varying Buffer Size (a) Infocom05, (b) Cambridge06

The impact of buffer size on delay is shown in Figure5. It can be seen that delivery delay increases as buffer size increases for all the 4 routing mechanisms. This is because when the buffer size increases, nodes can store and deliver more messages with longer delay, leading to the increased average delivery delay.

In Direct mechanism, each node only stores its own messages, cannot forward the messages to others with smaller delivery delay, so the average delivery delay is largest. The average delivery delay of CANCMDA is slightly larger than Smart and Multicent mechanisms. One reason is that the delivery ratio of CANCMDA is higher as in Figure 4, so more messages with longer delay are delivered, leading to the larger average delivery delay. Another reason is that in CANCMDA, a receiving node with high congestion degree may reject to forward the message to avoid it being dropped, and the message will be forwarded to another node with more free buffer but slightly lower delivery probability, leading to the larger average delivery delay.

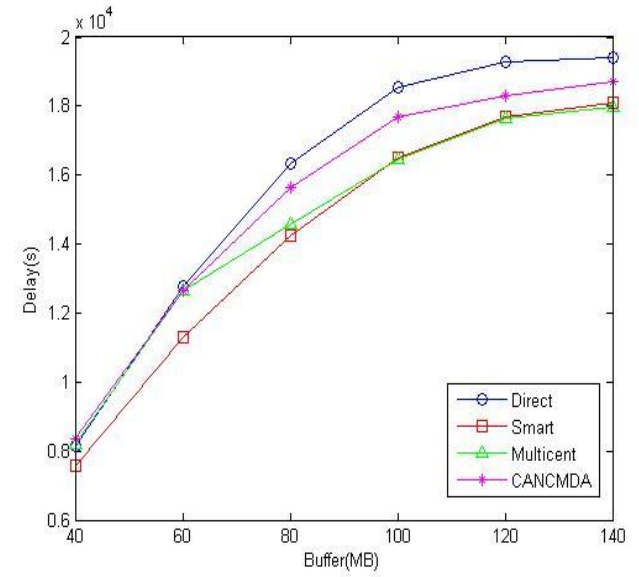

(a)

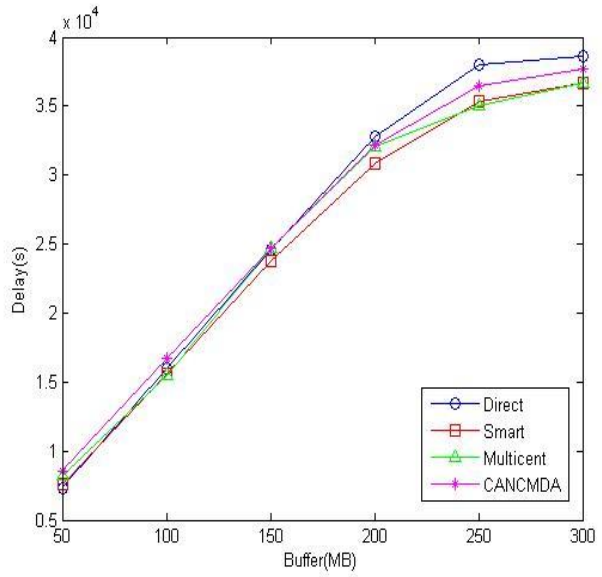

(b)

Figure 5. Delivery Delay for varying Buffer Size (a) Infocom05, (b) Cambridge06

The impact of buffer size on overhead ratio is shown in Figure 6. In Direct mechanism, each node only stores its own messages which are never forwarded by others, so the overhead ratio is always 1. For CANCMDA, Smart and Multicent mechanisms, the impact of buffer size has two aspects. On the one hand, as buffer size increases, nodes can 
get more forwarding chances, so the number of forwarding will increases, leading to the increase of overhead ratio. On the other hand, more messages are successfully delivered, leading to the decrease of overhead ratio. When the first impact outweighs the second one, then the overhead ratio increases. Otherwise the overhead ratio decreases.

The overhead ratios of Smart and Multicent are much higher than CANCMDA, this is because many messages are forwarded to the nodes with higher congestion degree through many hops, but finally dropped due to the node congestion. These invalid forwardings will increase the overhead ratios of Smart and Multicent mechanisms. In addition, from theorem 1 , we can see that if a message is traded successfully when $k$ is $1 / 2$, it has to satisfy the condition that $V_{\text {buy }}-V_{\text {sell }} \geq 1 / 4$. The difference of $V_{\text {buy }}$ and $V_{\text {sell }}$ has to be greater than or equal to the threshold of $1 / 4$, will also decreases the overhead ratio of CANCMDA. From above, we know that CANCMDA can achieve a higher message delivery ratio with lower overhead ratio, compared with other mechanisms, although having a slightly larger delay.

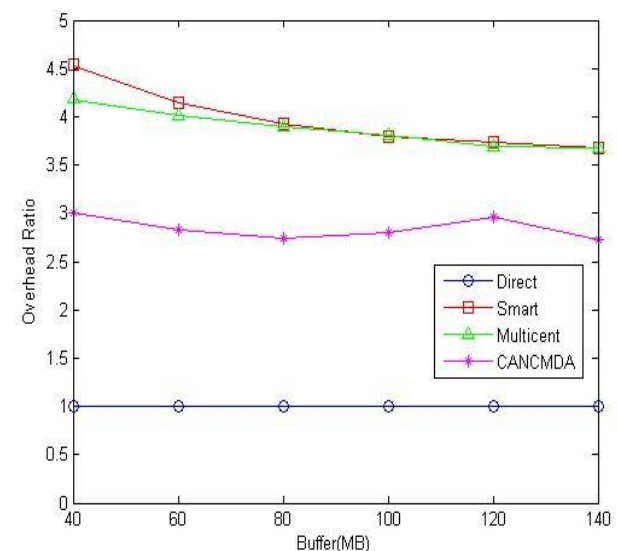

(a)

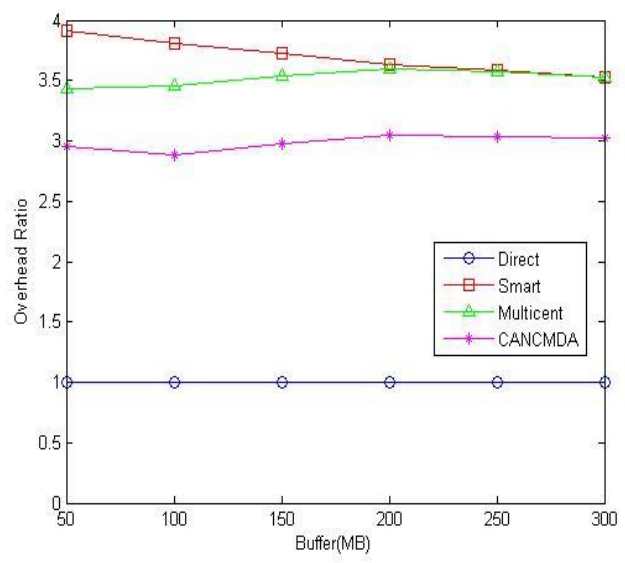

(b)

Figure 6. Overhead Ratio for Varying Buffer Size (a) Infocom05, (b) Cambridge06

5.2.2. Effect of TTL: The impact of TTL on delivery ratio is shown in Figure 7. The delivery ratio increases as the TTL increases for all the 4 routing mechanisms, because the message delivery chance is better with longer TTL. The delivery ratio of Direct scheme is lowest. This is because each node only stores its own messages, and cannot forward messages to other nodes with higher delivery probability. As a result, some messages are dropped due to the fullness of buffer or expiration of TTL. The delivery ratio of CANCMDA is higher than the Smart and Multicent mechanisms, because it can effectively avoid the node congestion. 


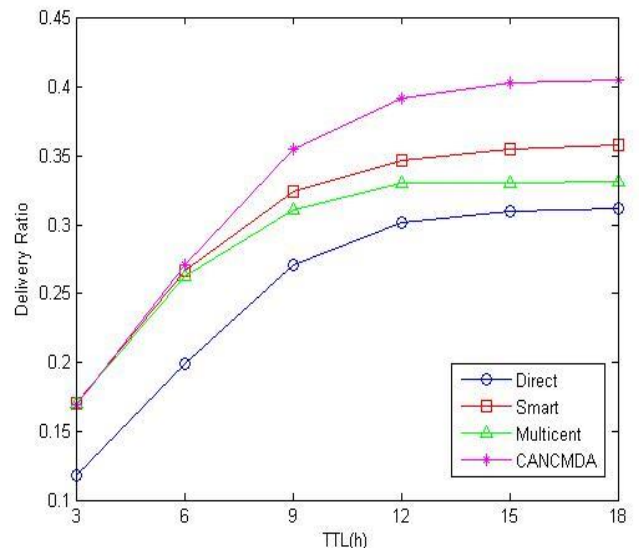

(a)

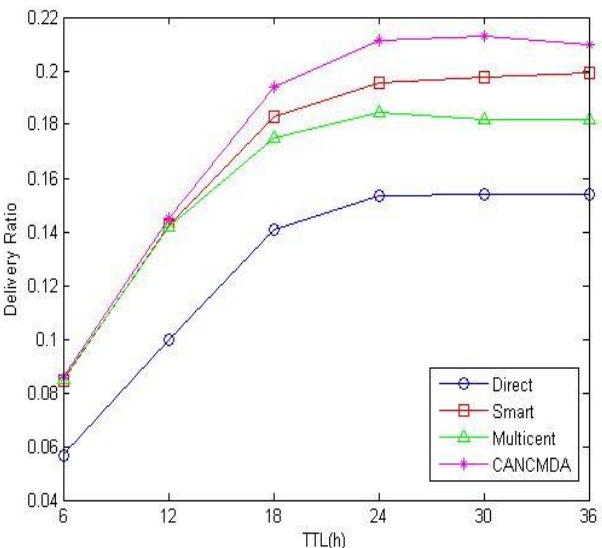

(b)

Figure 7. Delivery Ratio for Varying TTL (a) Infocom05, (b) Cambridge06

Figure 8 shows the impact of TTL on delay. It can be seen that delivery delay increases as TTL increases for 4 mechanisms. This is because as the TTL increases, nodes can store and deliver more messages with longer delay, leading to the increased average delivery delay. The delivery delay of Direct mechanism is largest, because node cannot forward the messages to others with smaller delivery delay. The average delivery delay of CANCMDA is larger because many messages are forwarded to the forwarding nodes with more free buffer but slightly lower delivery probability to avoid being dropped.

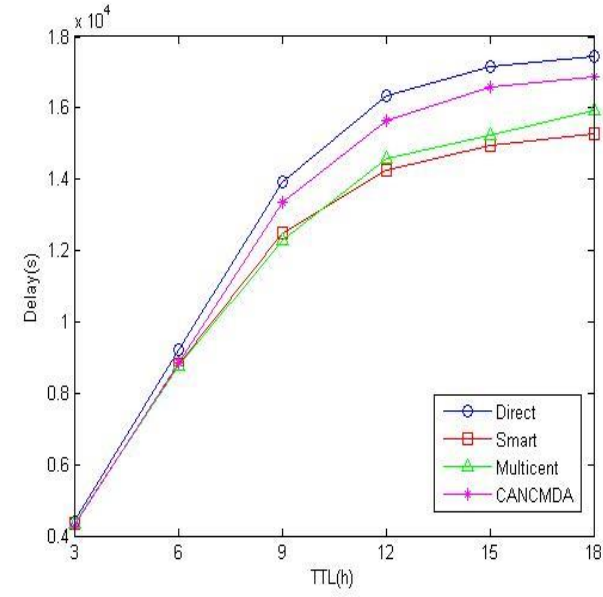

(a)

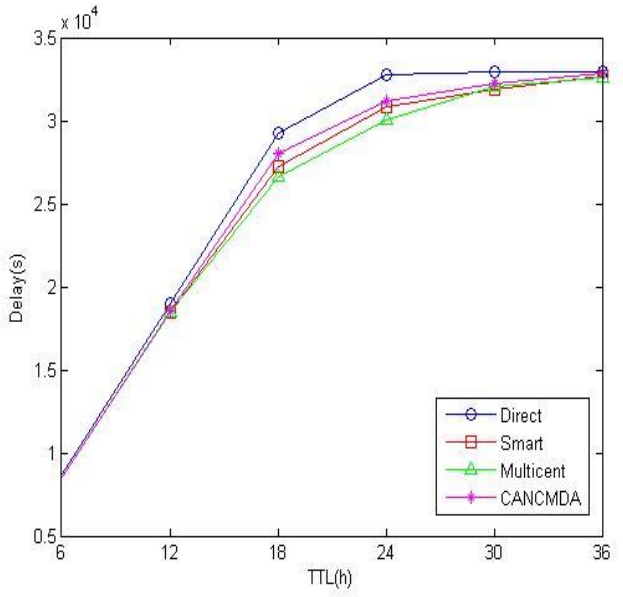

(b)

Figure 8. Delivery Delay for Varying TTL (a) Infocom05, (b) Cambridge06

Figure 9 shows the impact of TTL on overhead ratio. In Direct mechanism, the overhead ratio is always 1 , because the messages are not forwarded by other nodes. For the other 3 routing mechanisms, on the one hand, as message TTL increases, nodes can get more forwarding chance, leading to the increase of overhead ratio. On the other hand, more messages are successfully delivered, leading to the decrease of overhead ratio. The overhead ratio of CANCMDA mechanism is lower than Smart and Multicent mechanisms, due to the same reason as section 5.2.1. 


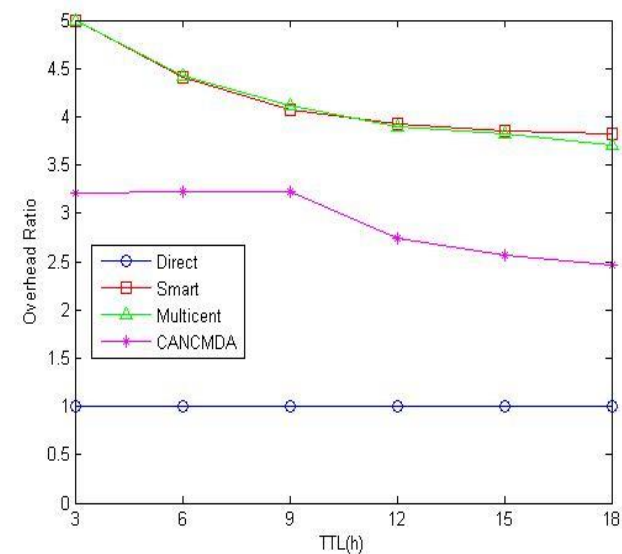

(a)

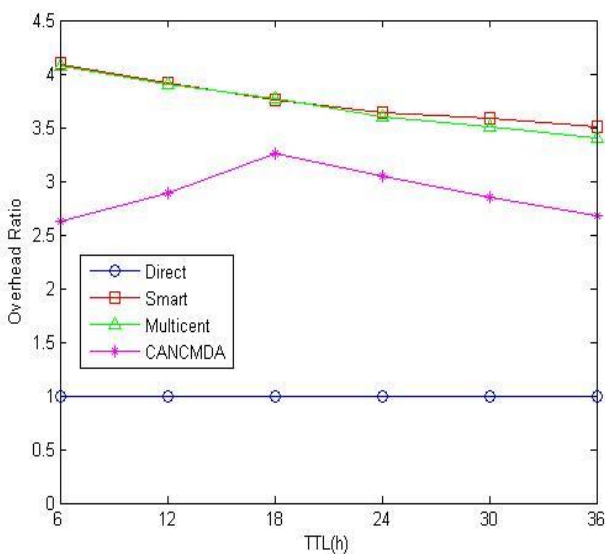

(b)

Figure 9. Overhead Ratio for Varying TTL (a) Infocom05, (b) Cambridge06

5.2.3. Effect of Message Generation Interval: In Figure10, it can be seen that the delivery ratio increases with the increase of message generation interval. When the message generation interval is larger, the total messages generated in the network will be smaller, and nodes can have enough resource of buffer and bandwidth to forward messages, so the delivery ratio is higher.

In Direct mechanism, node only forwards its own messages, so the delivery ratio is lowest. The delivery ratio of CANCMDA is much higher than Smart and Multicent mechanisms when the message generation interval is small. This is because when the message generation interval is small, more nodes will be congested, and CANCMDA mechanism can effectively reduce the impact of congestion. When the message generation interval is larger than 20, all nodes will have enough buffer, so CANCMDA, Smart and Multicent mechanisms have similar delivery ratios.

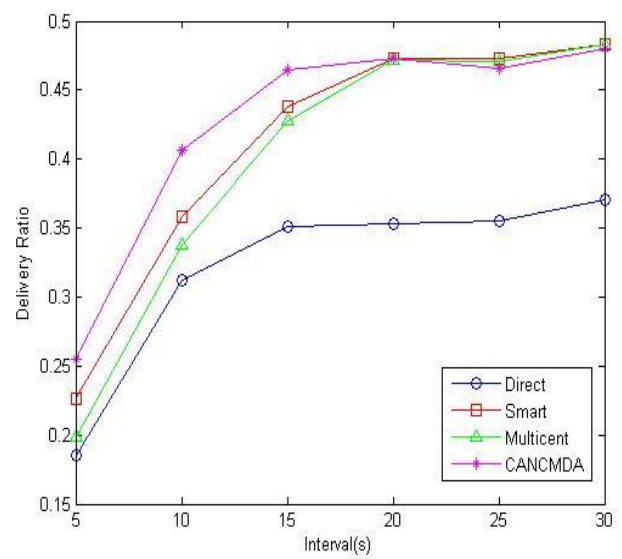

(a)

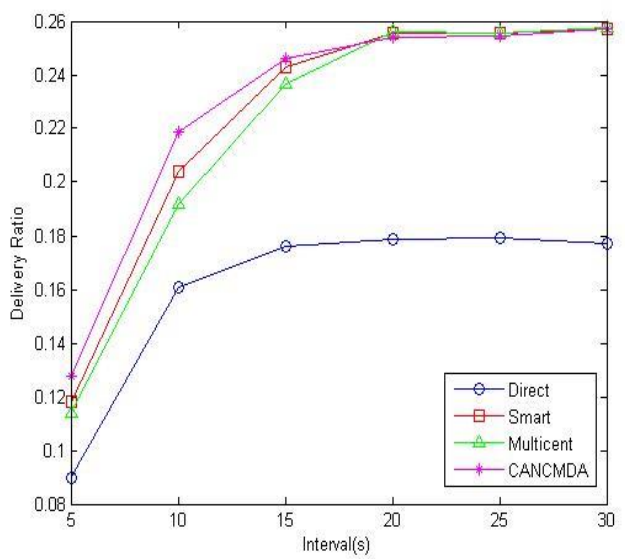

(b)

\section{Figure 10. Delivery Ratio for Varying Generation Interval (a) Infocom05, (b)Cambridge06}

Figure11 shows the impact of message generation interval on delay. It can be seen that delivery delay increases for all the 4 mechanisms as the message generation interval increases. When the message generation interval is small, more messages are generated in the network; so many messages with longer TTL will be dropped due to the limited buffer. Because the messages with longer delay can be forwarded when message 
generation interval is larger, the delivery delay will increase as the message generation interval increases. The average delivery delay of CANCMDA is larger than Smart and Multicent mechanisms, when the message generation interval is small. This is also because when the message generation interval is small, more messages are forwarded to the forwarding nodes with more free buffer but slightly lower delivery probability to avoid being dropped. When the message generation interval is larger, CANCMDA, Smart and Multicent mechanisms will have similar delivery delay, because nodes are not congested.

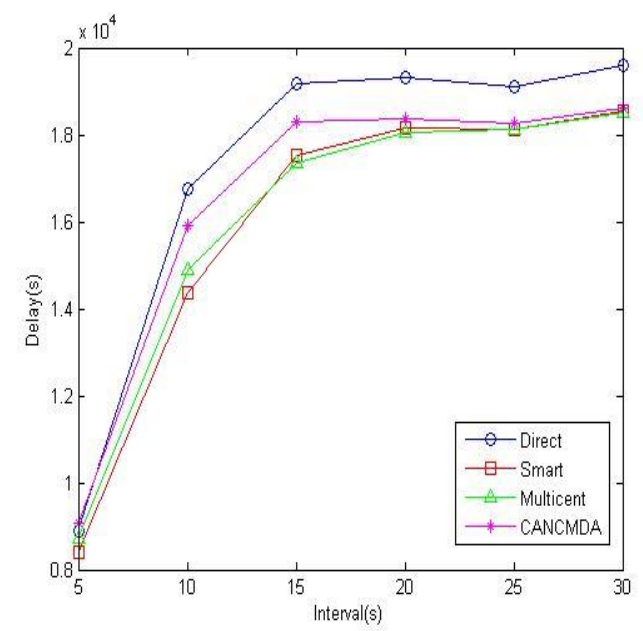

(a)

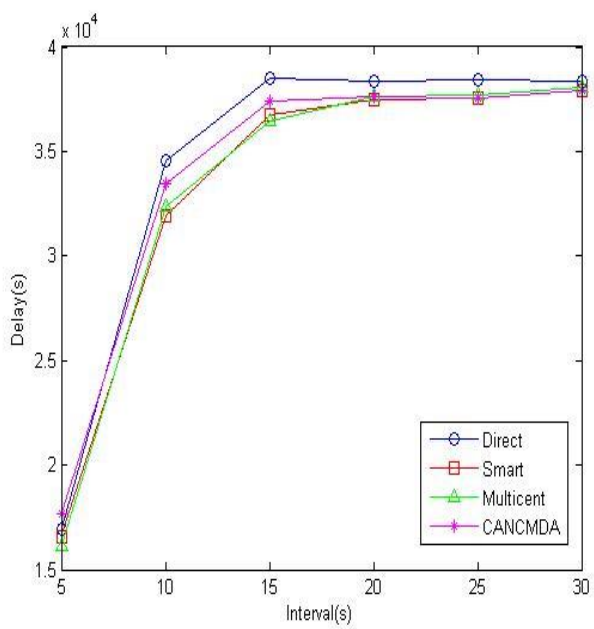

(b)

Figure 11. Delivery Delay for Varying Generation Interval (a) Infocom05, (b) Cambridge06

As shown in Figure 12, the overhead ratio of Direct mechanism is always 1. For the other 3 routing mechanisms, the impact has two aspects. On the one hand, as message generation interval increases, nodes can have enough bandwidth to forward messages, so the number of forwarding will increases, leading to the increase of overhead ratio. On the other hand, nodes will also have much buffer to deliver more messages, leading to the decrease of overhead ratio. When the first impact outweighs the second one, the overhead ratio increases, otherwise the overhead ratio decreases. The overhead ratio of CANCMDA mechanism is lower than Smart and Multicent mechanisms.

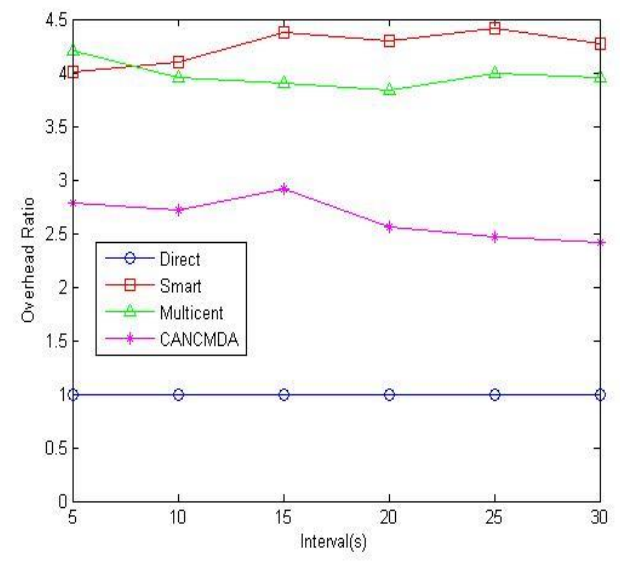

(a)

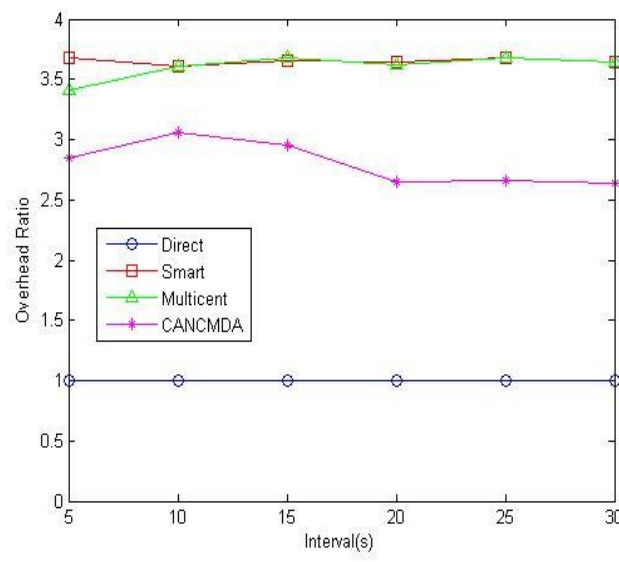

(b)

Figure 12. Overhead Ratio for Varying Generation Interval (a) Infocom05, (b) Cambridge06 
5.2.4. Cumulative Utility Comparison: As shown in Figure 13, in CANCMDA, most of the nodes can get more utilities than Smart and Multicent mechanisms. In Smart and Multicent, many messages are forwarded to the node with higher congestion degree, and being dropped due to the node congestion. In Smart, the total credits paid by the source node is divided by all the forwarding nodes, so when a message is dropped, all the forwarding nodes will lose their credits and get fewer utilities than the nodes in CANCMDA. In Multicent, the message destination nodes have to pay for the forwarding nodes even when a message is dropped, so the destination nodes will get fewer utilities due to the dropped message. CANCMDA mechanism can effectively reduce the number of messages dropped due to congestion, so the nodes can get more utilities than other mechanisms.

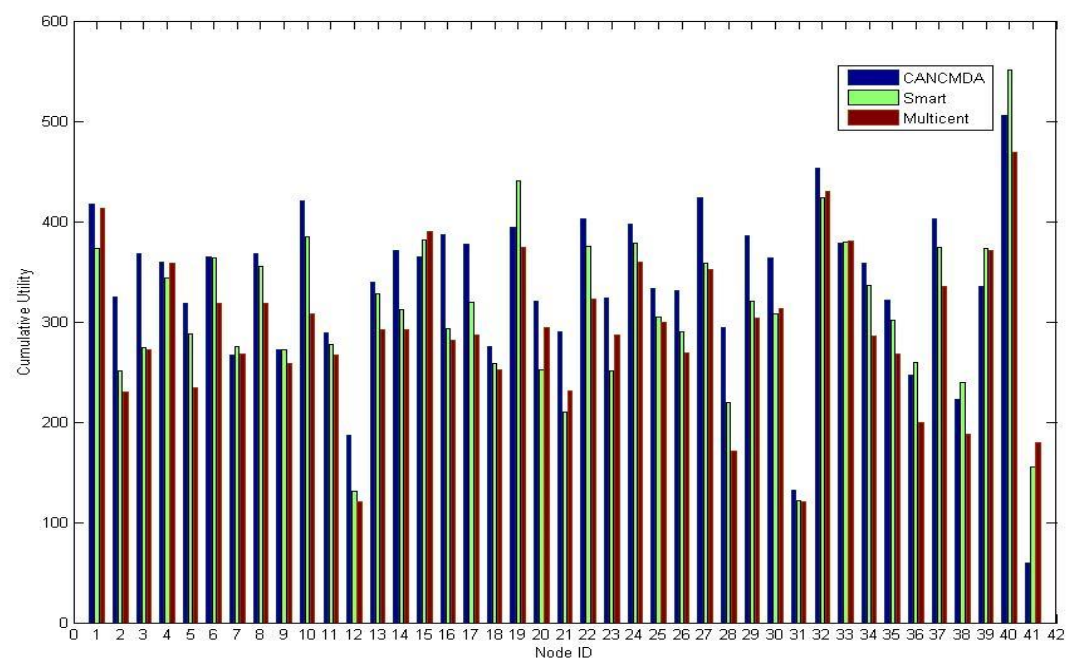

(a)

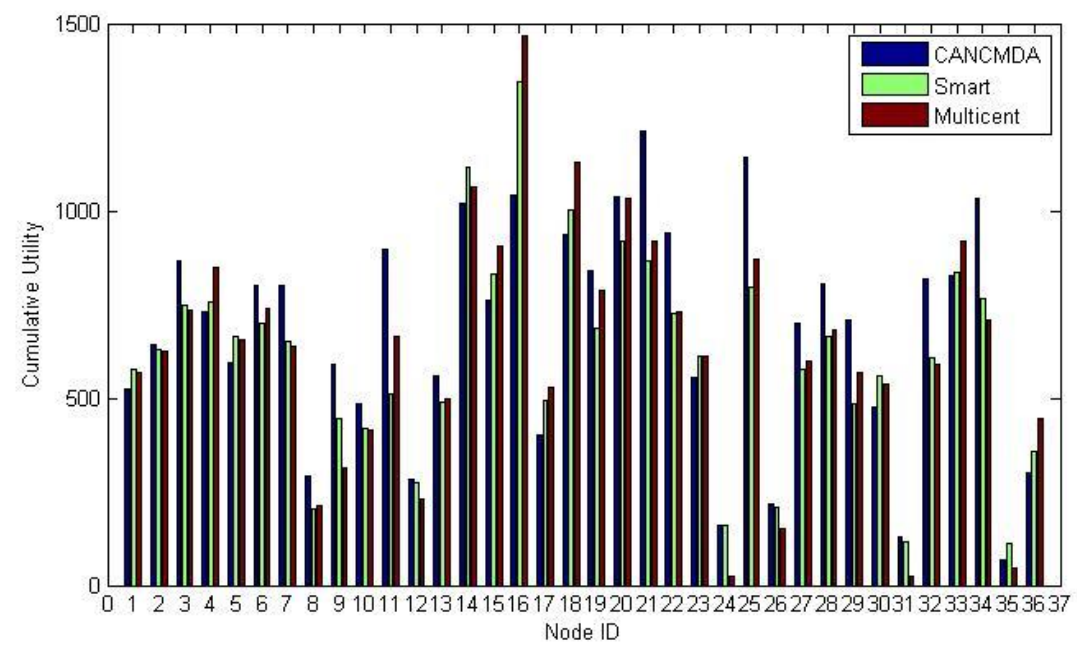

(b)

Figure 13. Cumulative Utility Comparison (a) Infocom05, (b)Cambridge06

\section{Conclusion and Recommendation}

In this paper, in order to effectively stimulate the selfish nodes of opportunistic networks to cooperatively forward messages when nodes are congested, a congestionaware node cooperation mechanism based on double auction, called CANCMDA is proposed. In CANCMDA, nodes determine the congestion degree according to the current 
free buffer size and message receiving speed. Nodes calculate the message expectation value by the delivery probability and congestion degree, then trade the messages based on double auction model, thereby achieving the cooperation. Trace driven experiments verify that CANCMDA can achieve a higher message delivery ratio with lower overhead ratio, compared with other mechanisms. Since this work focuses on the single copy routing, and our future direction is to explore an effective congestion aware node cooperation mechanism for the multi-copy routing and data dissemination in opportunistic networks.

\section{Acknowledgments}

This work is fully supported by Natural Science Foundation of Daqing Normal University (14zr21)

\section{References}

[1] L. Pelusi, A. Passarella and M. Conti, "Opportunistic Networking: Data Forwarding in Disconnected Mobile Ad Hoc Networks", IEEE Commun. Mag., vol. 44, no. 11, (2006), pp. 134-141.

[2] A. Vahdat and D. Becker, "Epidemic routing for partially-connected ad hoc networks", Technical Report CS-200006, Duke University, (2000).

[3] M. Grossglauser and D. N. C. Tse, "Mobility increases the capacity of ad hoc wireless networks", IEEE/ACM Transactions on Networking, vol. 10, no. 4, (2002), pp. 477-486.

[4] T. Spyropoulos, K. Psounis and CS. Raghavendra, "Efficient routing in intermittently connected mobile networks: the multiple-copy case", IEEE/ACM Transactions on Networking, vol. 16, no. 1, (2008), pp. 77-90.

[5] V. Erramilli, M. Crovella, A. Chaintreau and C. Diot, "Delegation Forwarding", Proc. of MobiHoc'08, Hong Kong, China, (2008) May 27-30.

[6] EM. DALY and M. HAAHR, "Social Network Analysis for Information Flow in Disconnected Delay Tolerant MANETs", IEEE Transactions on Mobile Computing, vol. 8, no.5, (2009), pp. 606-621.

[7] C. Boldrini, M. Conti, and A. Passarella, "Contentplace: social-aware data dissemination in opportunistic networks”, Proc. of ACM MSWiM'08, Vancouver, Canada, (2008).

[8] A. Panagakis, A. Vaios and I. Stavrakakis, "On the effects of cooperation in DTNs", Proc. of the 2nd International Conference on Communication System Software and Middleware and Workshops, Bangalore, India, (2007) January 7-12.

[9] Y. Li, G.L. Su, D.P. O. Wu, D.P. Jin, L. Su, and L.G. Zeng, "The impact of node selfishness on multicasting in delay tolerant networks", IEEE Transactions on Vehicular Technology, vol.60, no. 5, (2011), pp. 2224-2238.

[10] L. Buttyan, L. Dora, M. Felegyhazi and I. Vajda, "Barter trade improves message delivery in opportunistic networks", Ad Hoc Networks, vol.8, no.1, (2010), pp. 1-14.

[11] Y. Wang, M.C. Chuah and Y.Y. Chen, "Incentive based data sharing in delay tolerant mobile networks", IEEE Transactions on wireless communications, vol.13, no.1, (2014), pp. 370-381.

[12] R. X. Lu, X. D. Lin and Z. G. Shi, "IPAD: An Incentive and Privacy-Aware Data Dissemination Scheme in Opportunistic Networks", Proceedings of IEEE INFOCOM 2013, Turin, Italy, (2013).

[13] A. Mei and J. Stefa, "Give2Get:Forwarding in social mobile wireless networks of selfish individuals", IEEE Transactions on Dependable and Secure Computing, vol.9, no.4, (2012), pp. 569-582.

[14] B. B. Chen and M. C. Chan, "MobiCent:a credit-based incentive system for disruption tolerant network", IEEE Conference on Computer Communications (INFOCOM)2010, San Diego, USA, (2010) March 1519.

[15] H. L. Chen, and W. Lou, "Making Nodes Cooperative: A Secure Incentive Mechanism for Message Forwarding in DTNs", The 22nd International Conference on Computer Communications and Networks (ICCCN), Nassau, Bahamas, (2013) July 30-August 2.

[16] H. J. Zhu, X. D. Lin, R. X. Lu, Y. F. Fan and X. M. Shen, "SMART: A Secure Multi-Layer Credit based Incentive Scheme for Delay-Tolerant Networks", IEEE Transactions on Vehicular Technology, vol.58, no.8, (2009), pp. 4628-4239.

[17] K. Chen and H. Y. Shen, "Multicent:A Multifunctional Incentive Scheme Adaptive to Diverse Performance Objectives for DTN Routing", IEEE International Conference on Sensing, Communication, and Networking(SECON), New Orleans, USA, (2013).

[18] A. Grundy and M. Radenkovic, "Decongesting opportunistic social based forwarding", Proc. of the Seventh International Conference on Wireless On-demand Network Systems and Services (WONS), Kranjska Gora, Slovenia, (2010).

[19] J. Pujol, A. Toledo and P. Rodriguez, "Fair Routing in Delay Tolerant Networks", Proceedings of IEEE INFOCOM, Rio de Janeiro, Brazil, (2009).

[20] W. Gao, Q. Li, B. Zhao, and G.H. Cao, "Multicasting in delay tolerant networks: A social network perspective”, Proceedings of ACM MobiHoc'09, New Orleans, USA, (2009). 
[21] A. Krifa, C. Barakat and T. Spyropoulos, "Optimal Buffer Management Policies for Delay Tolerant Networks", The Fifth Annual IEEE Communications Society Conference on Sensor, Mesh and Ad Hoc Communications and Networks(SECON '08), California, USA, (2008).

[22] K. Chatterjee and W. Samuelson, "Bargaining under incomplete information", Operations Research, vol.31, no.9, (1983), pp. 835-851.

[23] M. J. Osborne, Editor, "An introduction to game theory", Oxford university Press, New York, (2009).

[24] A. Keranen, J. Ott and T. Karkkainen, "The ONE simulator for DTN protocol evaluation", Proceedings of the 2nd International Conference on Simulation Tools and Techniques, Rome,Italy,(2009).

[25] J. Scott, R. Gass, J Crowcroft, P. Hui, C. Diot and A. Chaintreau, CRAWDAD Trace Cambridge/ haggle/imote/infocom (v.2006-01-31), Downloaded from http://crawdad.cs.dartmouth.edu /cambridge/ haggle/imote/infocom, (2006).

[26] J. Leguay, A. Lindgren, J. Scott, T. Friedman, J. Crowcroft and P. Hui, CRAWDAD Trace upmc/content/ imote/cambridge(v.2006-11-17), from http://crawdad.cs.dartmouth.edu/upmc/ content/ imote/Cambridge, (2006).

[27] T. Karagiannis, J. Y. L. Boudec and M. Vojnovic, "Power Law and Exponential Decay of Inter Contact Times between Mobile Devices", IEEE Transactions on Mobile Computing, vol.9, no.10, (2010), pp. 1377-1390.

\section{Authors}

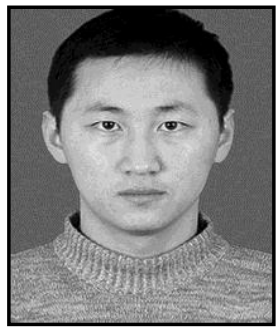

Qingfeng Jiang, he received his B.S. and M.S. degrees in computer science and technology from Heilongjiang University and Harbin Engineering University in 2005 and 2008 respectively. He is working towards his Ph.D. degree at Harbin Engineering University. His main research interests include delay tolerant networks and social networks.

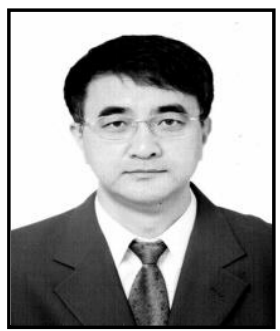

Chaoguang Men, he born in 1963. Professor and Ph. D. supervisor at Harbin Engineering University. Senior member of China Computer Federation. His main research interests include mobile computing and trusted computing.

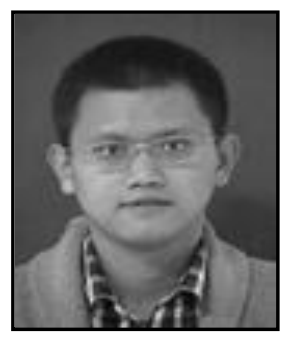

Zeyu Tian, he received his B.S. degree in computer science and technology from Harbin Engineering University in 2011. He is working towards his Ph.D. degree at Harbin Engineering University. His main research interests include mobile computing and trusted computing.

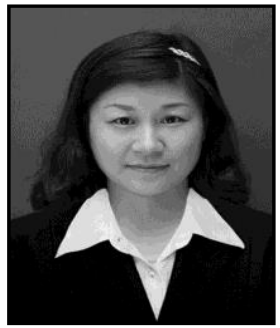

Meijuan Jia, she received his M.S. degrees in computer science and technology from Harbin Engineering University in 2006. She is working towards his Ph.D. degree at Harbin Engineering University. Her main research interests include mobile P2P networks and network security. 